Published in Potentiality, Entanglement and Passion-at-a-distance-Quantum Mechanical Studies for Abner Shimony, volume 2, edited by R. S. Cohen, M. Horne and J. Stachel, (Kluwer, Dordrecht, Holland 1997), p. 31-52.

\title{
CLASSICAL AND QUANTUM PHYSICAL GEOMETRY
}

\author{
JEEVA S. ANANDAN \\ Departments of Physics and Philosophy \\ University of South Carolina \\ Columbia, SC 29208, USA. \\ and \\ Sub-Faculty of Philosophy, University of Oxford \\ 10 Merton St., Oxford OX2 4JJ, UK.
}

\begin{abstract}
The task of creating a quantum theory of gravity is compared with Einstein's creation of a relativistic theory of gravity. The philosophical and physical foundations of this theory are briefly reviewed. The Ehlers-Pirani-Schild scheme of operationally determining the geometry of space-time, using freely falling classical particle trajectories, is done using operations in an infinitesimal neighborhood around each point. The study of the free fall of a quantum wave suggests a quantum principle of equivalence. The principle of general covariance is clarified. The sign change of a Fermion field when rotated by $2 \pi$ radians is used to argue for a quantum mechanical modification of space-time, which leads naturally to supersymmetry. A novel effect in quantum gravity due to the author is used to extend Einstein's hole argument to quantum gravity. This suggests a quantum principle of general covariance, according to which the fundamental laws of physics should be covariant under 'quantum diffeomorphisms'. This heuristic principle implies that space-time points have no invariant meaning in quantum gravity.
\end{abstract}

gr-qc/9712015 


\section{INTRODUCTION: PHYSICS AND PHILOSOPHY}

Many physicists and some philosophers hold the view that physics is an empirical science and that philosophers therefore have no place in it, except perhaps as historians. Abner Shimony has over the years opposed this narrow view of both physics and philosophy. One day he aptly summarized his distinguished roles in physics and philosophy by describing himself to me as a 'natural philosopher' and an 'experimental metaphysician'. He thereby emphasized the role of physics as natural philosophy and the relevance of philosophical principles to an experimental science such as physics. I was always in full agreement with this view. It is particularly relevant today because of the problem of quantizing gravity which has eluded the conventional methods of physicists and seems to call for a major paradigm shift. It seemed to me therefore that a fitting contribution to the Volume in Abner's honor would be to describe some work I have done towards applying philosophical principles to the task of quantizing gravity, which may be the most difficult and deepest of all the unsolved problems in theoretical physics today.

If we compare this task with the creation of quantum theory during the early part of this century, which led to a major paradigm shift, we find that there was a great deal of experimental evidence which physicists such as Planck, Einstein, Bohr, De Broglie, Heisenberg and Schrödinger could make use of in order to create quantum theory. This theory is so rich and counter intuitive that it would not have been possible for us, mere mortals, to have dreamt it without the constant guidance provided by experiments. This is a constant reminder to us that nature is much richer than our imagination. But there is no direct experimental evidence today on quantum gravitational phenomena which could guide us similarly in the construction of a quantum theory of gravity. So, we are left with the need to apply clever mathematical techniques, as in the case of superstring theory, or to apply philosophical principles, as in the present article, or both in order to create quantum gravity from almost nothing.

On the other hand, if we compare this task with another major paradigm shift of this century that accompanied the creation of general relativity, we find that the latter occurred with almost no guidance from experiment. This, I believe, was largely because of the genius 
of Einstein in judiciously applying philosophical principles and geometrical concepts to Newtonian gravity and special relativity, which led to the discovery of the deeper theory of general relativity which contained the first two theories as approximations. In section 1, I shall briefly describe this and argue in favor of following in Einstein's footsteps again.

\section{RELATIVIZING AND QUANTIZING GRAVITY}

After the discovery of special relativity by Lorentz, Poincare, and Einstein, there was the problem of "relativizing gravity", analogous to the problem of "quantizing gravity" which exists today. It was clear that Newtonian gravity was incompatible with special relativity and it was necessary to replace it with a relativistic theory of gravity. While several attempts were made to do this, Einstein succeeded in constructing such a theory

because he used i) the geometrical reformulation of special relativity by Minkowski, and ii) the operational approach of asking what may be learned by probing gravity using classical particles.

An important ingredient in (i) was Einstein's realization that the times in the different inertial frames, $t$ and $t^{\prime}$, in the Lorentz transformation were on the same footing. This made the Lorentz group of transformations a true symmetry of physics. Minkowki then constructed a space- time geometry by means of the metric that is invariant under the Poincare group of tranformations that is generated by the Lorentz transformations and translations acting on space-time. So, the interpretation Einstein gave to special relativity, whose basic equations were already known to Lorentz and Poincare, was crucial to the subsequent work of Minkwoski. It enabled Einstein to get rid of the three dimensional ether, and thereby pave the way for the introduction of the four dimensional 'ether', called space-time, by Minkowski.

By means of (ii), Einstein concluded that the aspect of Newtonian gravity which should be retained when this theory is modified is the equivalence principle. This principle is compatible with special relativity locally. This may be seen from the physical formulation of the strong equivalence principle according to which in the Einstein elevator that is freely falling in a gravitational field the laws of special relativity are approximately valid. But 
this principle allowed for the modification of special relativity to incorporate gravity as curvature of space-time.

Today we find that general relativity, the beautiful theory of gravity which Einstein discovered in this way, is incompatible with quantum theory. Can we then adopt a similar approach? This would mean that we should use 1) a geometrical reformulation of quantum theory, and 2) an operational approach of asking what may be learned by probing gravity using quantum particles.

As for (1), the possibility of using group elements as 'distances' in quantum theory, analogous to space-time distances in classical physics, was studied previously [1]. For a particular quantum system, the corresponding representations of these group elements may be used to relate points of the projective Hilbert space, i.e. the set of rays of the Hilbert space, which is the quantum generalization of the classical phase space [2]. Recent work on protective observation of the quantum state has shown that the points of the projective Hilbert space are real, in the sense that they could be observed by measurements on an individual system, instead of using an ensemble of identical systems [3].

As for (2), the question is whether the motion of a quantum system in a gravitational field enables us to identify the aspect of general relativity which must be preserved when this theory is replaced by a quantum theory of gravity, i.e. the quantum analog of the equivalence principle. I shall formulate such a principle, in this article.

In section 2, I shall review the classical equivalence principle and its use by Ehlers, Pirani and Schild (EPS) to determine the geometry of space-time from the trajectories of freely falling particles. I shall then provide a new formulation of the equivalence principle, in section 3 , in terms of the symmetry group acting in the first order infinitesimal neighborhood around each point. This modified equivalence principle is simpler and leads to the geometry more naturally than the EPS scheme. Also, it shows the connection between the different structures studied by EPS. Moreover, the EPS scheme breaks down when we go to quantum theory because the particles do not have trajectories (except in the Bohm interpretation of quantum theory in which the trajectories assigned to the particles are for the most part unobservable and therefore cannot be used to obtain the geometry). But the modified equivalence principle has a smooth transition to quantum theory. 
This will be shown in section 4 , where the objective will be to do the quantum mechanical version of the EPS scheme, i.e. to determine the geometry using wave motion instead of particle motion. A quantum weak equivalence principle and a quantum strong equivalence principle will be formulated. It may be noted that Einstein's equivalence principle, which he discovered in 1908, was largely a philosophical princple until the mathematical construction of general relativity. Similarly, the present quantum equivalence principles are largely philosophical, and would probably remain so until the construction of quantum gravity.

The principle of general covariance used by Einstein in his discovery of general relativity is studied in detail in section 5. The role of coordinate systems and symmetries is clarified. Einstein's hole argument is examined and the distinction between passive and active transformations is abolished.

In section 6, a novel effect due to the quantum superposition of two geometries on the wave function of a test particle is described. This effect is invariant under a quantum diffeomorphism that transforms different geometries differently. This freedom suggests that the points of space-time have no invariant meaning. So, there seems to be a need to get rid of the four dimensional 'ether', namely space-time, in order to incorporate the quantum diffeomorphism symmetry into quantum gravity. The covariance of the laws of physics under these quantum diffeomorphisms is formulated as a new principle of quantum general covariance.

\section{THE CLASSICAL EQUIVALENCE PRINCIPLE} AND THE EHLERS-PIRANI-SCHILD SCHEME

The classical weak equivalence principle (WEP), due to Galileo and Einstein has two aspects to it: In a space-time manifold with a pure gravitational field, a) the possible motions of all freely falling test particles are the same, and b) at any point $p$ in space-time, there exists a neighborhood $U(p)$ of $p$ and a coordinate system $\left\{x^{\mu}, \mu=0,1,2,3\right\}$, such that the trajectories of every freely falling test particle through $p$ satisfies [4]

$$
\frac{d^{2} x^{\mu}}{d \lambda^{2}}=0
$$


at $p$ for a suitable parameter $\lambda$ along the trajectory. This is the local form of the law of inertia and the above coordinate system is said to be locally inertial at $p$. The condition (b) is a special property of the gravitational field, not shared by any other field. For example, in an electromagnetic field test particles with the same charge to mass ratio would satisfy (a) but not (b). (The Lorentz 4-force is proportional to the electromagnetic field strength which, being a tensor, cannot be coordinate transformed away unlike the connection coefficients.)

Using (b), for massive and massless particles, it was shown by Ehlers, Pirani and Schild (EPS) [4], based on the earlier work of Weyl, that there exists an affine connection $\omega$ such that the trajectories of freely falling test particles are affinely parametrized geodesics with respect to it. I shall now present their arguments more clearly by means of operations in an infinitesimal neighborhood around each point, instead of using differential equations. The use of this neighborhood, which will be defined shortly, also will pave the way for an improved version of the classical equivalence principle by means of the symmetry group in this neighborhood, in section 3. The latter principle will be seen to have a smooth transition into quantum physics, unlike the equivalence principle as formulated by Galileo, Einstein or EPS.

Suppose $\epsilon=\frac{d}{L}$, where $d \sim$ linear dimensions of $U(p)$ and $L \sim$ radius of curvature obtained from the curvature components of this connection, all lengths being measured in the above coordinate system, and we can neglect second orders in $\epsilon$. Such a neighborhood will be called a first order infinitesimal neighborhood of $p$, and denoted by $U_{\epsilon}(p)$. Using the geodesic deviation equation, it may be shown that the velocities of the freely falling test particles in $U_{\epsilon}(p)$ are constant in an appropriately chosen coordinate system. This is a stronger form of the WEP than its usual statement given above, and will be called the modified classical weak equivalence principle. It is valid in Newtonian gravity as well as Einsteinian gravity.

Let us now look at the different geometrical structures that arise in $U_{\epsilon}(p)$ directly from the motions of particles, instead of assuming an a priori metric as in the above analysis.

Specifying the unparametrized geodesics in $U_{\epsilon}(p)$ gives it a projective geometry. Now the trajectories of massive freely falling particles are time-like geodesics. But the collection 
of such trajectories that pass through a given point contain as their boundary, the collection of null geodesics at that point. The tangent vectors to these null geodesics constitute the null cone at that point. Specification of the null cone at each point in space- time is the same as specifying the conformal structure of space- time.

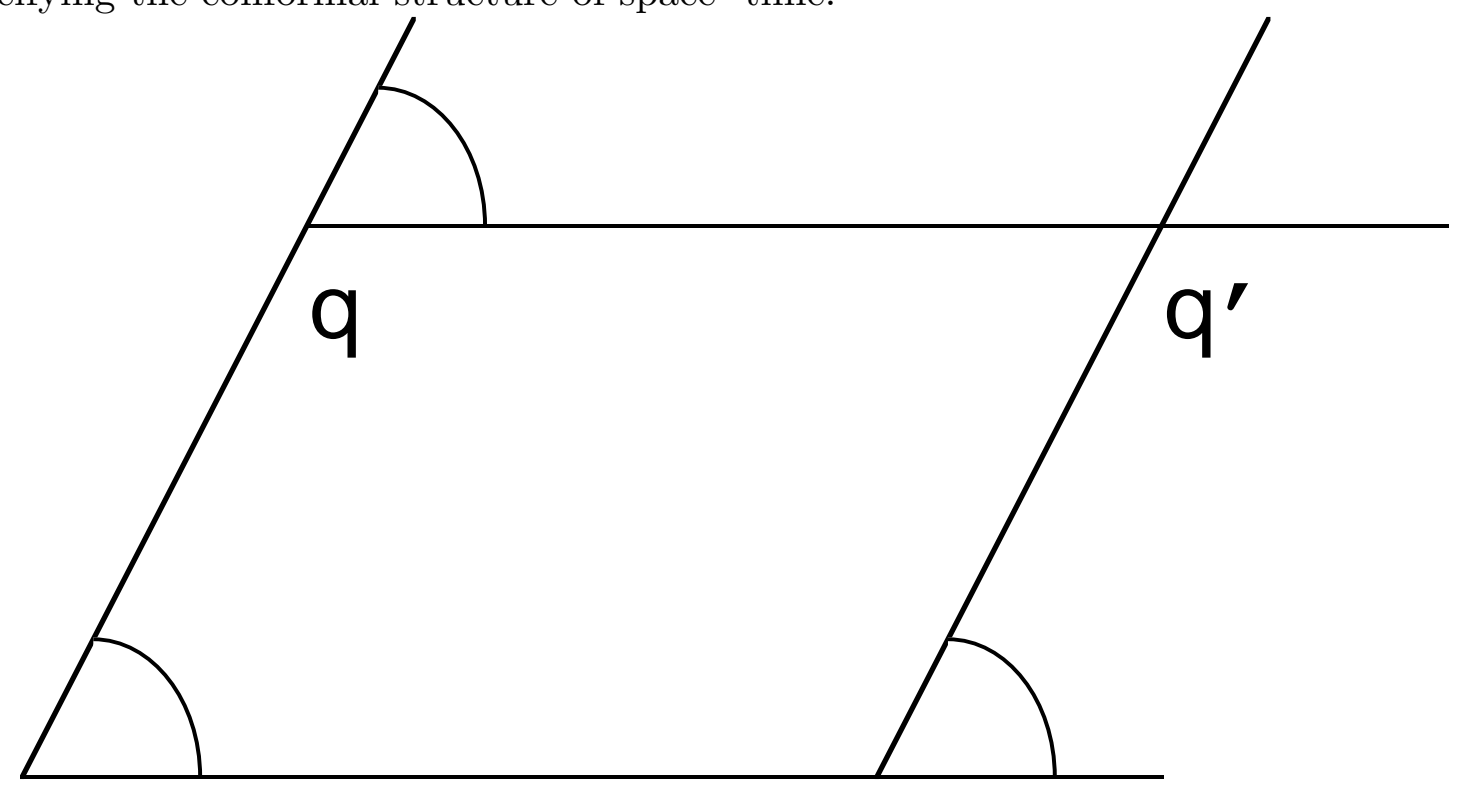

Figure 1. Detgrmination of the affine structure using the projegtive and conformal stuctures. The projective structure determines the lines and the conformal sturcture determines the equality of the angles indicated in the infinitesimal parallelogram $p p^{\prime} q^{\prime} q$. Then $p^{\prime} q^{\prime}$ may be regarded as the prallel transport of $p q$, which gives rise to the affine stucture.

The projective structure determines the "straight lines", or simply "lines" (the preferred curves of the projective structure) and the conformal structure determines angles in $U_{\epsilon}(p)$. Using these two concepts, an infinitesimal parallelogram may be constructed in $U_{\epsilon}(p)$ as follows: Let $p^{\prime}$ and $q$ be two points that are in $U_{\epsilon}(p)$ and distinct from $p$. There exist two unique preferred curves of the projective structure ( "lines" in $U_{\epsilon}(p)$ ) passing through $p, p^{\prime}$ and $p, q$ (Fig. 1). Let $V$ be the two dimensional vector space spanned by the tangent vectors to these two curves at $p$. The "lines" through $p$ that have the vectors in $V$ as tangent vectors form a two dimensional surface in $U_{\epsilon}(p)$ which will be called a "plane". Choose the unique point $q^{\prime}$ on this plane such that the line segment $p^{\prime} q^{\prime}$ makes the same angle as $p q$ with $p p^{\prime}$, and $q q^{\prime}$ makes the same angle as $p p^{\prime}$ with $p q$, as determined by the conformal structure. Then $q q^{\prime}$ will be said to be parallel to $p p^{\prime}$, and $p^{\prime} q^{\prime}$ will be said to be 
parallel to $p q$. Therefore, $p p^{\prime} q^{\prime} q$ is an infinitesimal parallelogram. It is emphasized that this construction does not use a metric. We now have an affine geometry in $U_{\epsilon}(p)$, because an affine geometry is a projective geometry together with the concept of parallelism. It is possible now to parallel transport a vector along an arbitrary curve as follows: Given two infinitesimally separated points $p$ and $p^{\prime}$ on the curve such that $p^{\prime}$ is inside $U_{\epsilon}(p)$, construct an infinitesimal parallelogram $p p^{\prime} q^{\prime} q$, with the direction of $p q$ being arbitrary, using the projective and conformal structures, as described in the previous paragraph. Then $p^{\prime} q^{\prime}$ is the parallel transport of $p q$. Then by suitable rescaling, any tangent vector at $p$ in the direction of $p q$ may be parallel transported to $q$ to be in the direction of $q q^{\prime}$. Note that this prescription fixes both the direction and the length of the transported vector as it should be under parallel transport, but without requiring a metric do so. Now that parallel transport for an infinitesimal displacement is known, it is possible to parallel transport an arbitrary vector along the entire curve, which is arbitrary except that it is piecewise differentiable. This defines an affine connection.

It may be noted that this affine connection is torsion free. This is because in the presence of torsion infinitesimal parallelograms do not close in general. Whereas the above affine connection is defined so that infinitesimal parallelograms always do close.

The affine connection together with the conformal structure is called a Weyl structure. In a Weyl space-time, it is possible to compare the lengths of two measuring rods at a given space-time point using the conformal structure. Also, it is possible to parallel transport either of them, using the affine connecton, so that the rod remains the 'same' during this process. (Cf. the opposite sides $p q$ and $p^{\prime} q^{\prime}$ of the above infinitesimal parallelogram, which are equivalent with respect to the affine geometry and therefore the 'same'.) But when this rod is parallel transported around a closed curve it would in general undergo a rotation (Lorentz transformation) and an elongation or contraction compared to the rod that was left at the original point.

The Lorentz transformation of a 4-vector under parallel transport around a closed curve is due to the space-time curvature which represents the gravitational field. Weyl tried to identify the change of length of the vector as being due to the electromagnetic field. This shows a lack of operationalism in Weyl's approach to the electromagnetic 
field, unlike Einstein's approach to the gravitational field via the equivalence principle obtained by probing the gravitational field with a classical particle. If we characterize the electromagnetic field by what it does to a charged probe that is used to measure the field, we find that the field does not cause any change in length. The field exerts forces on a classical charged particle, and it produces a phase factor on the wave function of a quantum particle. I shall deal with the latter aspect in more detail in section 4.

There is no experimental evidence at all for the above mentioned change in length postulated by Weyl with or without the electromagnetic field. It is therefore reasonable to suppose that space-time is Riemannian, i.e. it is a special case of Weyl space- time in which a vector parallel transported around a closed curve may come back rotated but without any change of length. EPS make this as an additional postulate in order to obtain the Riemannian structure of space-time.

\section{THE MODIFIED STRONG EQUIVALENCE PRINCIPLE}

There are two shortcomings in the EPS scheme, described in section 2. First, by using freely falling particle trajectories that satisfy the equivalence principle, EPS obtain naturally the Weyl structure and not the Riemannian structure. The additional postulate they make to obtain Riemannian geometry is ad hoc and is not suggested naturally by the operational procedure they adopted. Secondly, they consider several geometrical stuctures, and the connection between them appear mysterious. This seems to call for a simpler and more unifying principle.

I shall now give a new formulation of the equivalence principle, which does not have these shortcomings. The formulations of WEPs, given in section 2, may be stated using only an affine connection and do not require a metric. In $U_{\epsilon}$, the affine structure defined by this connection has as its symmetry group the affine group $A(4)$ that is generated by the general linear transformations and translations in a 4 dimensional real vector space. In the non relativistic limit, as the null cones 'flatten', $A(4)$ remains the symmetry group.

When the translational subgroup of $A(4)$ acts on a given point, the orbits are geodesics, which are the trajectories of freely falling particles. These curves satisfy the condition 
(2.1), and fulfill the usual formulation of the equivalence principle. Physically speaking, the translational invariance which exists in the absence of external forces implies, via Noether's theorem, the conservation of energy-momentum. Satisfying (2.1) is due to the local constancy of the energy-momentum of the freely falling particle. These preferred curves define the projective structure. So, we see here the 3 -fold connection between the symmetry group, the geometry and the physics, in this particular case of the equivalence principle.

In classical physics, the interactions between the particles restrict the symmetry group in $U_{\epsilon}$ to the inhomogeneous Galilei group (non relativistic physics), or the Poincare group $P$ (relativistic physics), which are both subgroups of $A(4)$. The existence of this residual symmetry group in $U_{\epsilon}$ is a form of the classical strong equivalence principle (SEP) valid for relativistic and non relativistic gravity. I shall call this the modified classical SEP.

In this way, non flat space-time geometry may also in some sense be brought into the frame-work of Felix Klein's Erlanger program according to which a geometry is determined as the set of properties invariant under a symmetry group [1].

As mentioned above, the translational subgroup acting on $U_{\epsilon}(p)$ of the Poincare group determines the projective structure. The Lorentz subgroup leaves invariant the null cone at each point $p$ and therefore determines the conformal structure. So, the relationship between these two structures can now be understood algebraically in terms of the relationship between these two subgroups of the Poincare group.

The modified SEP also can be extended to wave motion. The particle trajectories which EPS used are obtained in the geometric optics limit of the quantum wave. In this limit, the information contained in the phase of the wave function is lost. Once this phase information is restored, the compatibility between the metric and the connection which EPS introduced, in order to specialize the Weyl structure resulting from the projective and conformal structures to the Riemannian structure, naturally follows.

The metric compatibility follows from the fact that in quantum theory there is a natural frequency $\omega$ associated with a mass $m$ given by

$$
m c^{2}=\hbar \omega
$$


which acts as a clock. Using the distances along time-like curves measured by this clock and light signals, whose motion is determined by the conformal structure, it is possible to determine the metric, as shown long ago by Synge. And $m^{2}=\eta^{a b} P_{a} P_{b}$ is a Casimir operator of the Poincare group which means that it commutes with every element of this group. It will be shown in the next section that this implies that space- time is Riemannian.

\section{THE QUANTUM EQUIVALENCE PRINCIPLE}

What fundamental aspects about the gravitational field may be learned if it is probed with quantum particles, instead of with classical particles as in the above treatment? It was shown that the evolution of a freely falling wave function is given, in the WKB approximation, by the action on the initial wave function by the operator [5]

$$
\Phi_{\gamma}=\operatorname{Pexp}\left[-i \int_{\gamma} \Gamma_{\mu} d x^{\mu}\right]
$$

where

$$
\Gamma_{\mu}=\theta_{\mu}{ }^{a} P_{a}+\frac{1}{2} \omega_{\mu}^{a}{ }_{b} M^{b}{ }_{a} .
$$

which will be called the gravitational phase operator. Here the energy-momentum operators $P_{a}$ and the angular momentum operators $M_{a}^{b}, a, b=0,1,2,3$ generate the covering group of the Poincare group $\tilde{P}$ that is a semi-direct product of $S L(2, C)$ and space-time translations $R(4)$. The fact that mass $m$ is a good quantum number in curved space-time and $m^{2}$ is a Casimir operator of $P$ already suggests that $P$ is relevant in the presence of gravity.

For every space-time point $p$, let $H_{\epsilon}(p)$ be the Hilbert space of wave functions in $U_{\epsilon}(p)$ in which $\tilde{P}$ acts. Owing to the linearity of the action of (4.1), it determines also the evolution of any freely falling wave packet which can be expanded as a linear combination of WKB wave functions, provided the size of the wave packet is small compared to the radius of curvature, i. e. it is contained primarily inside $U_{\epsilon}$ at each point along $\gamma$ which may be chosen to be along the center of the wave packet. This will be called the quantum weak equivalence principle, because (4.1) is a Poincare group element independent of the freely falling wave packet. In this respect, it is like the classical WEP according to which the affine connection determined is independent of the test particle used. 
In quantum physics, because the wave packet must necessarily have some spread, the WEP cannot be formulated by particle trajectories as in conditions (a) and (b) in section 3 , and it is necessary to use at least the neighborhood $U_{\epsilon}$. Indeed (4.1) was obtained [5] using the Klein-Gordon [6] and Dirac equations [7] which are covariant under $\tilde{P}$ in $U_{\epsilon}$. So, in quantum physics there is a close connection between the WEP, as formulated above, and SEP according to which $\tilde{P}$ is the symmetry group of all laws of physics in $U_{\epsilon}$. It is well known that (a) cannot be valid in quantum physics, because the motions of wave functions depend on their masses [8]. But the modified classical WEP and the classical SEP formulated in sections 2 and 3 have the advantage that they have a smooth transition to quantum physics.

The above approximate concepts may be made mathematically precise as follows: Each neighborhood $U_{\epsilon}(p)$ may be identified with the tangent space at $p$ regarded as an affine space. The motions of freely falling test particles relate affine spaces associated with two neighboring points by a linear transformation and a translation, generated by $P_{a}$. This gives a natural connection on the affine bundle [9] over spacetime which is a principal fiber bundle with $A(4)$ as the structure group. This is the connection used above to express the modified classical WEP. The quantum WEP requires the Poincare subbundle with $\tilde{P}$ (to admit Fermions) as the structure group. Then (4.2) defines a connection in this principal fiber bundle. The gravitational phase operator (4.1) parallel transports with respect to this connection along the curve $\gamma$. The above Hilbert space bundle, that is the union of $H_{\epsilon}(p)$ for all space-time points $p$, is a vector bundle associated to this principal fiber bundle with a connection that is the representation (4.2) in this Hilbert space.

The curvature of the above connection is the Poincare Lie algebra valued 2-form

$$
F=d \Gamma+\Gamma \wedge \Gamma=Q^{a} P_{a}+\frac{1}{2} R_{b}^{a} M_{a}^{b},
$$

where, on using) and the Lie algebra of the Poincare group,

$$
Q^{a}=d \theta^{a}+\omega_{b}^{a} \wedge \theta^{b}, R_{b}^{a}=d \omega_{b}^{a}+\omega^{a}{ }_{c} \wedge \omega^{c}{ }_{b} .
$$

which are called respectively the torsion and the linear curvature. If the wave equation used to obtain (4.1) did not contain torsion, then the torsion in (4.3), of course, is also 
zero. However, the above modified classical WEP and the quantum WEP make it natural to have torsion and suggest that if the torsion is zero then there should be a good physical reason for it.

Suppose $\gamma$ is a closed curve. Then (4.1) is a holonomy transformation determined by the above affine connection. The importance of (4.1) may also seen by comparing it with the corresponding phase factor for electromagnetism:

$$
\exp \left(-i \int e A_{\mu} d x^{\mu}\right)
$$

which is an element of the $\mathrm{U}(1)$ gauge group, where $A_{\mu}$ is the 4 - vector potential. It was pointed out by Yang [10] that the importance of (2), which appears in the wave function of a particle with charge $e$, was recognized by Schrdinger [11] in 1922, in his study of Weyl's gauge theory, four years before he introduced the wave function. The question then arises whether (4.1) is similarly the "shadow" of some important yet to be discovered concept in quantum gravity. In any event, the analogy between (4.1) and (4.5) implies that gravity may be regarded as a gauge field in the spirit of Chen Ning Yang's integral formulation of gauge field [12].

Evaluating (4.1) for a closed curve spanning an infinitesimal area $d \sigma^{\mu \nu}$ gives

$$
\Phi_{\gamma}=1+\frac{i}{2}\left(Q_{\mu \nu}{ }^{a} P a+\frac{1}{2} R_{\mu \nu}^{a b} M_{b a}\right) d \sigma^{\mu \nu}
$$

where $Q^{a}$ and $R_{b}^{a}$ are respectively the torsion and linear curvature. While this makes it natural to introduce torsion into gravity, there have been no experimental tests so far to test the presence of torsion, or to put an upper limit on it.

But motivated by this result, I obtained as an exact solution the most general stationary cosmic string containing torsion [13], by solving the simplest generalization of Einstein's gravitational field equations to include torsion. This is the gravitational analog of the solenoid in electromagnetism which produces the Aharonov-Bohm (AB) effect [14]. The gravitational AB effect due to the phase factor (1) is considerably richer [15]. Also, the solution I obtained is of interest in astrophysics because of the possible role of cosmic strings in galaxy formation, which is an important problem in explaining the observed universe. 
It follows from (4.6) that in the absence of gravity in a simply connected region (4.1) is path independent. I shall take the equivalent statement that the path dependence of (4.1) implies gravity as the definition of the gravitational field even when the region is not simply connected. This definition makes the converse of this statement also valid. So, by probing gravity using quantum mechanical systems, without paying any attention to gauge fields, gravity may be obtained naturally as a Poincare gauge field.

Comparing now the present scheme with the EPS scheme, which uses particle motion to obtain the geometry of space-time, the present scheme, which uses wave motion instead, does not need to bring in anything external in order to obtain compatibility of metric and connection. To see this consider two beams which go along two different paths from one space-time point $A$ to another point $B$. The metric along each beam is determined by the Casimir operator. But (4.1), which determines the evolution of each beam, being an element of the Poincare group, commutes with the Casimir operator $m^{2}$. Therefore this Casimir operator remains the same as it is transported along each beam and hence, using the phase as a clock, the two identical clocks along the two beams are in agreement after the two beams meet.

An advantage of this point of view is that it also provides a unified description of gravity and gauge fields. If a wave function is interacting not only with the gravitational field but also other gauge fields, then its propagation in the WKB approximation is given by the action of an operator of the form (4.1) with

$$
\Gamma_{\mu}=\theta_{\mu}{ }^{a} P_{a}+\frac{1}{2} \omega_{\mu}^{a}{ }_{b} M^{b}{ }_{a}+A_{\mu}{ }^{j} T_{j}
$$

where $A_{\mu}{ }^{j}$ is the Yang-Mills vector potential and $T_{j}$ generate the gauge group $G$. So, (4.1) now is an element of the entire symmetry group, namely $\tilde{P} \times G$. Thus, unlike the classical WEP, the quantum WEP naturally extends to incorporate all gauge fields.

The above fact that the observation of all the fundamental interactions in nature is via elements of the symmetry group suggest a symmetry ontology. By this I mean that the elements of symmetry group are observable and therefore real. Moreover, the observables such as energy, momentum, angular momentum and charge, which are usually observed in quantum theory are some of the generators of the above symmetry group. Observation 
always requires interaction between the observed system and the apparatus. Ultimately, these interactions are mediated by gravity and gauge fields, which act on the matter fields through elements of the symmetry group. I therefore postulate that the only observables which can actually be observed are formed from the generators of symmetry group, which according to our current understanding of physics are generators of $\tilde{P} \times G$.

\section{CLASSICAL GENERAL COVARIANCE AND SPACE-TIME POINTS}

It was mentioned in section 1 that historical lessons from Einstein's relativization of gravity may be useful in the quantization of gravity. An important step in Einstein's journey towards general relativity, apart from the principle of equivalence already dealt with in sections 2.2-4, was his discovery of the principle of general covariance. Unlike the principle of equivalence whose importance, in suggesting the incorporation of gravity as curvature of space-time, was realized by Einstein as early as 1908, he did not feel comfortable with general covariance. Indeed he first rejected this principle in 1913 on the basis of his 'hole argument' which will be discussed later in this section. This delayed the construction of general relativity by two years. His eventual resolution of the hole argument in favor of accepting general covariance enabled him to write down soon afterwards the gravitational field equations which overthrew Newtonian gravity, after its reign of two and a half centuries.

In view of the great confusion which surrounded and still surrounds the principle of general covariance and the role of coordinate systems among many physicists and philosophers, including Einstein, it would be worthwhile to examine it in some detail, as I shall do now. In the next section, I shall formulate a new principle of quantum general covariance which I hope would be similarly useful in constructing a quantum theory of gravity.

In special relativity, it was believed that there was a real, objective space-time manifold, the set of space-time points with a four dimensional Euclidean topology and differentiable structure. This manifold is simply connected and is endowed with an a priori, fixed Minkowski metric. I shall call this the absolute Minkowski metric, to distinguish it from 
other Minkowski metrics on this manifold which will be discussed shortly. Its curvature

$$
R_{\mu \nu}^{\rho}{ }_{\sigma}=0
$$

everywhere. Conversely, the metric of Lorentzian signature that satisfies (5.1) everywhere in a simply connected space-time must necessarily be a Minkowski metric. However, the latter metric is not unique. This is readily seen from the fact that (5.1) is generally covariant, by which is meant that any diffeomorphism on space-time leaves the form of (5.1) unchanged. Therefore, given any metric that is a solution of (5.1), any diffeomorphism on space-time maps it into another metric which is also a solution of (5.1). Or, to put it even more trivially, the new metric is isometric, by definition, to the old metric and therefore describes the same flat geometry. It follows that this space-time has an infinite number of Minkowski metrics which are all solutions of (5.1).

Hence, according to the present ontology of space-time, giving the absolute Minkowski metric on space-time has more information than giving (5.1) because it singles out one of the infinitely many possible Minkowski metrics, that are solutions of (5.1), as the actual metric. Later, I shall use Einstein's hole argument to change this ontology, which will lead to the rejection of the above statement in italics. Then the latter statement would become analogous to Newton's attempt to introduce an absolute space even though Newton's laws are covariant under Galilei boosts.

The space-time manifold, together with the absolute Minkowski metric on it will be denoted by $M$. On $M$, which is our 'arena', there are also matter fields, classical or quantum. These are 'painted' on $M$ by which I mean that they are appropriately differentiable functions of $M$ that do not distort the (Minkowski) geometry of $M$. (I.e. treating special relativity as a limiting case of general relativity, the back reaction of the matter fields on the space-time geometry is neglected). I shall denote $M$ together with the matter fields on it that satisfy the laws of physics, and which are just as real as the points of $M$, by $M^{*}$. So, $M^{*}$ is a mathematical representation of a possible universe.

To focus our ideas, consider the classical electromagnetic field $F_{\mu \nu}$ which satisfies the Maxwell's equations

$$
F_{; \nu}^{\mu \nu}=j^{\mu}, F_{[\mu \nu, \rho]}=0,
$$


where $j^{\mu}$ is the current density. Here (5.2) is written in an arbitrary coordinate system, with $; \nu$ representing the covariant derivative using the Christoffel connection formed from the metric coefficients in this coordinate system. Such general coordinates are sometimes called curvilinear coordinates to distinguish them from the Minkowski coordinates in which the metric coefficients take the usual Minkowski form $\eta_{\mu \nu}$. They are useful for solving particular problems. E.g. if there is spherical symmetry then it is convenient to use spherical polar coordinates.

Two types of transformation in $M$ may be distinguished. One is a passive transformation, which is a coordinate transformation amounting to a mere relabeling of the points of $M$. The other is an active transformation that is a diffeomorphism of $M$ onto itself, while the coordinate system is kept fixed. Both transformations leave (5.1) covariant. But singling out the absolute metric makes them very different. The active transformations which leave the absolute metric on $M$ invariant is the Poincare group generated by the Lorentz transformations and the space-time translations. But passive transformations consist of the much larger group of diffeomorphisms. This is because the specification of a coordinate system requires only the differentiable structure, and therefore a change of coordinates need to keep only the differentiable structure invariant.

The transformations on $M$ also transform appropriately the matter fields on $M^{*}$, which are tensor or spinor fields on $M$. To specify spinor fields on $M$, it is necessary also to define a 'vierbein' field which is a differentiable choice of local Lorentz frames. Operationally, the value of a spinor field at a space-time point is what would be observed by an observer using the local Lorentz frame that is the value of the vierbein field at the same point. Therefore, transformation of the vierbein field must be specified, in addition to the coordinate transformation, in order to determine the transformation of the spinor field. But for each Minkowski coordinate system, it is convenient to choose the corresponding vierbein field to be the coordinate basis. Then the transformations between the Minkoski coordinate systems, consisting of the Poincare group of transformations, automatically determine the transformations of the vierbein field, and hence of the spinor fields.

A physical process in $M^{*}$ is defined to be a collection of matter fields which satisfy all the laws of physics, given by equations such as (5.2). Then the principle of special 
relativity due to Einstein may be stated as follows: Given any physical process in $M^{*}$, its transform by an active Poincare transformation of $M$ is also a physical process. I emphasize that this formulation assumes an absolute space-time $M$ relative to which these transformations produce new configurations. Later on I shall give up this assumption, which would necessitate defining a symmetry transformation as keeping something fixed.

An active transformation of $M^{*}$ that transforms any physical process to another physical process will be called a symmetry of the laws of physics. It is easily shown that the set of symmetries form a group. Instead of first specifying a metric a priori in $M$ and requiring that the active transformations which leave it invariant are also symmetries of the laws of physics, we could start with the group $G$ of symmetries on $M^{*}$ and obtain the geometry as the set of properties invariant under $G$, in accordance with Klein's Erlanger program [1]. In fact, if we insist on the determination of the geometry operationally by means of physical processes, involving clocks, measuring rods, etc., the symmetries of the laws of physics must necessarily be the symmetries of the geometry. This led to the formulation of the principle of physical geometry in ref. [1] according to which the symmetry group $P$ of the laws of physics is strictly the same as the symmetry group $G$ of the geometry:

$$
P=G \text {. }
$$

From this point of view, we cannot make the above philosophical distinction between $M$ that contains fixed absolute geometrical structures and $M^{*}$ that contains in addition variable, dynamical structures.

In general relativity, which superseded special relativity, (5.1) is replaced by the field equations

$$
G^{\mu \nu}=8 \pi T^{\mu \nu},
$$

where $G^{\mu \nu}$ is the Einstein tensor formed from the curvature tensor in (5.1) and $T^{\mu \nu}$ is the energy- momentum tensor. Then (5.4) imply via the Bianchi identities that

$$
T_{; \nu}^{\mu \nu}=0,
$$

which represents local conservation of energy-momentum. In addition, a prescription should be given for determining $T^{\mu \nu}$ as a function of the matter fields in a generally covariant manner. Then (5.5) incorporates the equations of motion for the matter fields [16]. This 
is because the local conservation of energy-momentum in the interactions between matter fields largely determine their evolution. For example, if $T^{\mu \nu}$ is the energy-momentum tensor of the electromagnetic field then (5.5) incorporates (5.2).

So, a remarkable feature of the Einstein-Hilbert field equations (5.4) is that, together with the prescription for $T^{\mu \nu}$ as a functional of the matter fields, it incorporates all the laws of classical physics, because it implies (5.5). On the other hand, without this prescription, (5.4) is a tautology because it is then merely a definition of $T^{\mu \nu}$ which automatically satisfies (5.5).

Another remarkable feature of (5.4) which makes general relativity fundamentally different from any previous classical theory is that it makes the metric dynamical. By a field being dynamical here I mean that the field is not given a priori as a fixed or absolute object but is determined by solving the field equations. In special relativity, the objects of $M$ were fixed, while the additional objects of $M^{*}$ were dynamical. Similarly, we may make a preliminary division between these two types of objects in general relativity: Define by $M_{G}$, the space-time manifold with only its topological and differentiable structures. Let $M_{G}^{*}$ denote $M_{G}$ together with the metric and matter fields.

Now, (5.4) is generally covariant. So, the symmetries of (5.4) are the group of diffeomorphisms on $M_{G}^{*}$. This is in accordance with the above mentioned principle of physical geometry because the differentiable structure is the geometry that is invariant under the group of diffeomorphisms.

We may now distinguish between two types of general covariance. First, it is possible to cast any law of physics in a generally covariant form, which is due to coordinates being labels. This reflects the unavoidable freedom to change the coordinate system by any passive diffeomorphism, without changing any of the structures whether they are absolute or dynamical. I shall call this passive general covariance. This exists in special and general relativity. Second, there is the just described symmetry group of active diffeomorphisms in general relativity due to the space-time metric becoming dynamical. I shall call this active general covariance. This is analogous to the local gauge symmetry in gauge theories which is related to the dynamical nature of gauge fields.

In the light of the above analysis, let us now examine Einstein's resolution of the 
hole argument [17]. In 1913, Einstein and Grossmann [18] considered the determination of the gravitational field inside a hole in some known matter distribution by solving the gravitational field equations. If these field equations have active general covariance, then there are an infinite number of solutions inside the hole, which are isometrically related by diffeomorphisms. I shall call these geometries Einstein copies.

This is unlike the case of the determination of the electromagnetic field in special relativity as described above. The electromagnetic field is uniquely determined inside a hole of some known charge distribution in Minkowski space-time $M$ by solving Maxwell's equations. Uniqueness here means that the field is obtained as a unique function of the space-time points inside the hole. But as mentioned earlier, these points may always be relableled by doing a coordinate transformation which is reflected in the passive general covariance of (5.1).

The Einstein copies may however be regarded as different representations of the same objective physical geometry. This follows if a space-time point inside the hole is defined operationally as the intersection of the world-lines of two material particles, or geometrically by the distances along geodesics joining this point to material points on the boundary of the hole. Under a diffeomorphism, such a point in one Einstein copy is mapped to a unique point in another Einstein copy. Both points may then be regarded as different representations of the same physical space-time point or an event.

So, we may identify as the same universe the equivalence class of all Einstein copies of $M_{G}^{*}$ that are related by active diffeomorphisms. This abolishes the distinction between passive and active general covariance. Because, after the above identification has been made, the physical points of space-time remain the same under passive and active diffeomorphisms, both of which represent equally a mere change of labels.

Alternatively, as some philosophers have done, it is possible to regard the real universe $M_{G}^{*}$ as embedded in an uncountably infinite set of mathematical copies of $M_{G}^{*}$. The active diffeomorphism freedom then enables us to move around this infinite set only one of which is real. This approach may also be taken in gauge fields by treating only one of the infinite equivalence class of gauge potentials related by gauge transformations to be the real gauge field. 
But I reject the latter approach for the following three reasons: First, as already mentioned in connection with the hole argument, the operational determination of spacetime points by matter fields forces us to identify all these different copies of $M_{G}^{*}$ because the corresponding points of $M_{G}^{*}$ are determined by the same procedure. Second, the latter view requires that we distinguish between active and passive transformations, whereas in differential geometry there is no distinction. Also, eqs. (5.1-13) are mathematically covariant equally under passive and active transformations. So, the latter approach does violence to the close connection between geometry and physics which the present paper regards as desirable. Finally, the above identification is necessitated by the use of Occam's razor, first because it reduces the uncountably infinite Einstein copies to the original and second because it abolishes the distinction between passive and active general covariance as shown above.

To recapitulate, there are two important philosophical points in the above analysis of general relativity. First, $M_{G}$ is used as the arena for the dynamical fields including the metric. Second, the identification of the Einstein copies of $M_{G}^{*}$ into a single space, which I shall call $\bar{M}_{G}^{*}$, makes the passive and active transformations the same. Both these aspects may be carried over to special relativity, which may be regarded as the limiting case of general relativity corresponding to weak gravitational sources and the special case of a simply connected space-time topology. Then $M_{G}$, instead of $M$, is the arena and the metric on $M_{G}$ may be obtained by solving (5.1), which is just as generally covariant as (5.4). But the different copies of $M_{G}$ together with the metrics on them that are solutions of (5.1), but which are diffeomorphically related, are now identified to be the same Minkowski space-time. This replaces $M$, and will be denoted by $\bar{M}$.

The question now arises as to what the symmetries are in special relativity. Geometrically speaking, the answer depends on which structures are kept fixed. If the Minkowski metric is regarded as an absolute structure, then the symmetries are the transformations which leave the geometry of $\bar{M}$ invariant, namely the Poincare group of transformations. If only the differentiable structure is kept fixed then the symmetries are the ones which leave the geometry of $\bar{M}_{G}$ invariant, namely the group of diffeomorphisms. A symmetry transformation is now redefined to assume implicitly that the transformation is being per- 
formed relative to something which is kept fixed, which we may take to be a frame [19]. This may be made precise by defining the transformation physically on $\bar{M}_{G}{ }^{*}$ by specifying the frame by matter fields as some sort of a grid. Then the transformation gives a different $\bar{M}_{G}{ }^{{ }^{\prime}}$ which cannot be identified with $\bar{M}_{G}{ }^{*}$ as was done above with the Einstein copies. The transformation being a symmetry means that $\bar{M}_{G}{ }^{{ }^{\prime}}$ is allowed by the laws of physics.

Instead of keeping the frame fixed and transforming the physical process, we may perform a passive symmetry transformation by keeping the physical process fixed and

transforming the matter fields constituting the frame (grid). But the new $\bar{M}_{G}{ }^{* \prime}$ obtained this way is an Einstein copy of $\bar{M}_{G}{ }^{*^{\prime}}$. So, the two should be identified according to our principle. This abolishes the distinction between active and passive symmetry transformations.

An example of the symmetry depending on which structure is kept fixed are the Maxwell's equations (5.2) in special relativity. They are generally covariant with respect to the differentiable structure, but are Poincare covariant in relation to the Minkowski metric.

As another example, consider the formulation of Newtonian mechanics as generally covariant Lagrange's or Hamilton's equations. This general covariance is a source of confusion among physicists and philosophers and needs to be clarified. With respect to the symplectic structure in phase space, the latter equations are generally covariant. Because all coordinate transformations (diffeomorphisms) of space leave the symplectic structure in phase space invariant. But with resepect to the Euclidean metric of Newtonian physics (an absolute as opposed to a dynamical structure) this general covariance is purely formal because the diffeomorphisms, in general, do not leave the Euclidean metric invariant. It is the Euclidean group on space, or the ten parameter Galilean group on space- time, which leaves the entire geometry invariant and therefore is the symmetry in this case.

\section{FERMIONIC NATURE OF SPACE-TIME}

In sections 2 and 5, classical matter fields were used to establish the ontology of the space-time manifold in classical physics. But in quantum theory, there are also Fermionic 
fields which have no classical analog. The question arises as to what kind of geometry is obtained if we apply the same philososphical and physical principles used to construct the classical physical geometry, above, now to the Fermionic fields. I shall present in this section joint work I did with Yakir Aharonov which provides an answer to this question by enlarging space-time so that it has a fundamental Fermionic nature.

The discussion of the hole argument in section 5 strongly suggested that we must regard space-time points as having a relational meaning instead of an absolute meaning. (This is implicit in the above specification of the points inside the hole by their distances along geodesics from points on the boundary of the hole.) In this spirit, consider the relationship between two neighboring space- time points $A, B$. This may be specified by the connecting vector $\overrightarrow{A B}=\epsilon v$, where $\epsilon$ is infinitesimal and $v$ is a physically observable tangent vector at $A$. Then if the connecting vector is rotated about $A$ by $2 \pi$ radians in some 2-plane, $B$ will return to itself.

Consider now two points $A, B$ joined by a connecting spinor $\sqrt{\epsilon} \psi$. If this spinor is rotated by $2 \pi$ radians it returns with the sign changed. It was first thought that this sign change is not observable, in which case we should say that $B$ returns to itself as before. But Aharonov and Susskind [20] showed that this sign change is observable. So, we must conclude that $B$ returns to a different point $B^{\prime}$.

The necessity for regarding $B$ and $B^{\prime}$ as distinct points may also be seen from the following two arguments. As mentioned, when a spinor field is rotated by $2 \pi$ radians then it does not return to itself. If this field is defined on the usual space-time then rotating the space-time, instead, by $2 \pi$ radians would bring it back to itself. This would make active transformations different from passive transformations. But in section 5 it was argued that we should abolish the distinction between active and passive transformations. This could be accomplished now only if we allow for a passive transformation on space-time that would rotate $B$ to a distinct point $B^{\prime}$.

Moreover, according to the principle of physical geometry (5.3), the symmetries of the physics are the same as the symmetries of the geometry. Since the rotations by $2 \pi$ and $4 \pi$ radians are distinguishable for Fermionic fields, the same should be true of the corresponding rotations acting on space-time. So, again, space-time should be enlarged so 
that the latter two rotations acting on the enlarged space-time are distinct.

This distinction may be made by specifying the variable point $B$ with respect to the fixed point $A$ by means of of the connecting spinor $\psi$ introduced above. Now, $\psi e^{i \theta}$ varies continuously from $\psi$ to $-\psi$ as $\theta$ varies from 0 to $\pi$. Hence, this defines a continuous transformation from $B$ to $B^{\prime}$, which are connected to $A$ by the spinor. Indeed as $\theta$ varies from 0 to $2 \pi$, it is clear that each space-time point now moves around a circle, whose points cannot be classically distinguished. We postulate that this $U(1)$ group of transformations is a symmetry of the theory for the following reason. In quantum theory, continuous symmetries are more natural than they are in classical physics. This is because, if a given configuration is transformed into another configuration by a discrete symmetry, then in quantum theory we can form a continuum of linear superpositions of the two configurations permitting a continuous group of transformations that connect the original discrete symmetry to the identity.

We can turn this argument around in view of the symmetry ontology proposed at the end of section 4. We may regard the symmetry group to be ontologically prior to the linear structure of the Hilbert space. It may be that the linear structure in quantum mechanics is required in order that continuous symmetries can act on the space of states.

Denote the generator of the new $U(1)$ symmetry, above, by $R$. Then, $\exp (i \theta R)$ acts on the geometry as well as the matter fields. Thus, space-time is now fundamentally quantum mechanical. Consider the action of this group on a pair of Bosonic and Fermionic fields. When $\theta=\pi$, the corresponding transformation should introduce a relative minus sign between the two fields. If the initial state of this pair of fields is represented by the column vector $(1,1)^{T}$ then the action of $R$ on this pair of fields is represented by $\frac{1}{2} \sigma_{z}$, with $\sigma_{x}, \sigma_{y}, \sigma_{z}$ being the usual Pauli spin matrices.

But to distinguish between the initial state $(1,1)^{T}$ and the transformed state $i(1,-1)^{T}$, corresponding to $\theta=\pi$, it is necessary to have another observable, say $Q=\frac{1}{2} \sigma_{x}$, which has these two states as its eigenvectors. Then, according to the symmetry ontology of section 4 , the observable $Q$ should generate a symmetry. Now, $Q$ generates supersymmetry transformation between the Bosonic and Fermionic fields. The commutator of $R$ with $Q$ gives another supersymmetric generator $Q^{\prime}=\frac{1}{2} \sigma_{y}$. So, $Q, Q^{\prime}$ and $R$ generate an $S U(2)$ 
algebra.

We illustrate the above ideas now by means of a simple example of a pair of Bosonic and Fermionic degrees of freedom, which are generated from the vacuum by the creation operators $a^{\dagger}$ and $b^{\dagger}$, respectively. These operators satisify the commutation and anticommutation relations

$$
\left[a, a^{\dagger}\right]=1,\left\{b, b^{\dagger}\right\}=1, b^{2}=0=\left(b^{\dagger}\right)^{2}
$$

The Hamiltonian is

$$
H=E\left(a^{\dagger} a+b^{\dagger} b\right)
$$

where $E$ is a constant. Then, we may take

$$
Q=\frac{1}{2} N^{-\frac{1}{2}}\left(b^{\dagger} a+a^{\dagger} b\right), Q^{\prime}=\frac{i}{2} N^{-\frac{1}{2}}\left(b^{\dagger} a-a^{\dagger} b\right), R=-i\left[Q, Q^{\prime}\right]=-2 i Q Q^{\prime}
$$

where $N=a^{\dagger} a+b^{\dagger} b$. Then,

$$
Q^{2}=Q^{\prime 2}=R^{2}=\frac{1}{4} I,\left\{Q, Q^{\prime}\right\}=0
$$

where $I$ is the identity operator. Clearly, $Q$ and $Q^{\prime}$ generate supersymmetric transformations between Bosonic and Fermionic states. And, it is easily seen that $Q, Q^{\prime}$ and $R$ commute with $H$ and are therefore symmetries of this model. Also, $Q, Q^{\prime}$ and $R$ generate an $S U(2)$ group.

To conclude this section, the application of our general principles to the sign change of the Fermion field when it is rotated by $2 \pi$ radians has not only led naturally to supersymmetry, but also has given a new symmetry generated by $R$. The details of this work will be published elsewhere [21].

\section{QUANTUM GENERAL COVARIANCE}

It is a curious fact that most approaches to quantum gravity use the classical spacetime manifold as the arena, as in classical general relativity, but with quantum fields (including the metric fields) instead of classical fields defined on it as operator value functions. But in fact quantum mechanics is formulated in Hilbert space and it is not possible 
to determine the points of space-time using quantum mechanical states because of the uncertainty principle. Operationalists such as E. Wigner have used this to argue that space-time manifold is not meaningful in quantum theory. Moreover, since each gravitational field is associated with a space-time geometry, quantizing it makes this geometry indefinite. So, even the points of space-time may be indefinite. I shall now describe a new effect which I obtained in quantum gravity $[22,13]$ which suggests that this may be the case.

Suppose that the gravitational field of a string is quantized so that different geometries corresponding to different angular momenta of the string may be superposed. Each of these geometries is flat in any simply connected region outside the string. So, their separate effects on any given simply connected region $U$ would be like as if there is no graviational field. However if we put a test particle in the region $U$, and the string is observed to be in a superposition of different angular momentum states then the wave function of the test particle would be affected. Its intensity has a variation in position due to the superposed geometries even though each of them is flat.

This effect is surprising and novel from a physical and philosophical point of view. What concerns us here are only the philosophical aspects which I shall discuss now. The above effect depends on the relationship between the metric coefficients of the two superposed geometries in $U$, in the particular gauge in which the gravitational field is quantized. But since each of these two geometries has zero curvature, it is possible to have quantized in a gauge in which both metric coefficients are the same and have the Minkowski values in $U$. So, how could their superposition affect the wave function of the particle?

The pair of superposed geometries in the new quantum gauge are obtained from the pair of superposed geometries in the old quantum gauge by performing different diffeomorphisms on the two geometries. Since each diffeomorphism has no effect on the geometry, as discussed in section 5, we may expect that this transformation on the superposed geometries also would not affect any physical phenomenat. Indeed, careful analysis $[23,20]$ shows that in the new quantum gauge also the same effect occurs, although the mathematical analysis now is very different.

In general, if there is a quantum superposition of gravitational fields, by a quantum 
diffeomorphism, or simply a q- diffeomorphism, I mean performing different diffeomorphisms on the superposed gravitational fields. Then the above two quantum gauges are related by a q-diffeomorphism performed on the quantized gravitational field. Because in bringing both metric coefficients to the same form in $U$, it is necessary to perform two different diffeomorphisms on them. These two diffeomorphisms transform the space-time points in $U$ differently. Then, as already mentioned, the above mentioned effect is invariant under this q-diffeomorphism.

I postulate now that all physical effects are invariant under all q-diffeomorphisms. This suggests a generalization of the usual principle of general covariance for the classical gravitational field to the following principle of quantum general covariance in quantum gravity: The laws of physics should be covariant under q-diffeomorphisms.

On the other hand, the usual principle of general covariance requires covariance of the laws of physics under classical diffeomorphisms, or c-diffeomorphisms. A c-diffeomorphism is a diffeomorphism that is the same for all the superposed gravitational fields, and is thus a special case of a q-diffeomorphism. Therefore, the above principle of quantum general covariance generalizes the usual general covariance due to Einstein. Under a cdiffeomorphism, a given space-time point is mapped to the same space-time point for all of the geometries corresponding to the superposed gravitational fields. This is consistent with regarding the space-time manifold as real, i.e. a four dimensional ether. So, if we restrict to just c-diffeomorphism freedom, space-time may be regarded as objective and real, as already shown in section 5 .

But the space-time points associated with each of the superposed gravitational fields, which are defined above in a c-diffeomorphism invariant manner, transform differently under a q- diffeomorphism. This means that in quantum gravity space-time points have no invariant meaning. However, protective observation suggests that quantum states are real [3]. Consequently, the space-time manifold, which appears to be redundant, may be discarded, and we may deal directly with the quantum states of the gravitational field.

It is the quantum uncertainty in the gravitational field which makes points of spacetime meaningless. Should we quantize the set so that cardinality is itself is uncertain? Since I showed [1] that cardinality is a physical and geometrical property, it would seem 
reasonable to quantize it. But then the curve $\gamma$ in the gravitational phase operator (4.1) cannot be meaningfully defined as a curve in space-time. The resolution of this difficulty may be expected to lead us to a quantum theory of gravity that may be both operational and geometrical.

\section{CONCLUDING REMARKS}

The above analysis suggests a philosophical principle which may be schematically be expressed as

$$
\text { Ontology }=\text { Geometry }=\text { Physics. }
$$

The last equality has not been achieved yet by physicists because we do not have quantum gravity. But it is proposed here as a philosophical principle which should ultimately be satisfied by a physical theory.

In relation to the hole argument, described in section 5 , this principle implies that the points of space-time become real in classical physics in virtue of the geometrical relations between them. The operational determination of an event as an intersection of material world-lines (e.g. lightening striking the railway track a la Einstein) is a way of observing it. This is like, for example, observing temperature by a thermometer. There are many different thermometers which may be used, but the concept of temperature is independent of them and may be defined as the average kinetic energy of the molecules at a given location. Relating a concept to experiments does not deny the possibility of an intrinsic meaning to that concept.

But in quantum physics, space-time points are not meaningful [1]. This is particularly so when gravity is quantized, as argued in section 7, above. The symmetry ontology which was proposed in section 4 , however, suggests (8.1) by providing a link between ontology, geometry and physics, as I shall argue now. The symmetries of the laws of physics are universal in the sense that they are the same for all laws and for all physical systems governed by them. They are independent of the particular spaces on which they act depending on the particular systems. The symmetries should therefore be used to construct the geometry, which should be universal. The conserved quantities implied by 
these symmetries, via Noether's theorem, is the "stuff" of the universe, and may be called real. The interactions depend on these conserved quantitites and elements of the symmetry group as we saw in section 4. Symmetry is destiny.

The question then arises as to how space-time may be obtained from the symmetry group. I believe that this is due to a common property of all interactions which picks out a subgroup of the symmetry group, which may be called the isotropy group. Then space-time emerges as the coset space of the isotropy subgroup. This is true not only of classical space-time but also the quantum space-time introduced in section 6 . In relation to space-time, this common property may therefore be called the locality of the interactions. That all interactions should possess this common property suggests that they should all be unified into a single interaction.

In pursuing this new unified theory of all interactions, it may be worthwhile to keep in mind the following statement due to Einstein, paraphrased by Bergmann [24], which I learned from Abner Shimony: “... a systematization of the experimental facts is by itself not yet a physical theory and ... in many respects the theoretical physicist is a philosopher in workingman's clothes".

\section{ACKNOWLEDGEMENTS}

I thank H. R. Brown for useful discussions.

\section{REFERENCES}

[1] J. Anandan, Foundations of Physics, 10, 601 (1980).

[2] J. Anandan, Foundations of Physics, 21, 1265 (1991).

[3] Y. Aharonov, J. Anandan, and L. Vaidman, Phys. Rev. A 47, 4616 (1993); Y. Aharonov and L. Vaidman, Phys. Lett. A 178, 38 (1993); J. Anandan, Foundations of Physics Letters 6, 503 (1993).

[4] J. Ehlers, F. A. E. Pirani, and A. Schild, in Papers in Honour of J. L. Synge, edited by L. O'Raifeartaigh (Clarendon Press, Oxford 1972).

[5] J. Anandan in Quantum Theory and Gravitation, edited by A. R. Marlow (Academic 
Press, New York 1980), p. 157.

[6] J. Anandan, Phys. Rev. D 15, 1448 (1977).

[7] J. Anandan, Nuov. Cim. A 53, 221 (1979).

[8] D. Greenberger, Ann. Phys. 47, 116 (1986); D. Greenberger and A. W. Overhauser, Sci. Am. 242, 66 (1980).

[9] S. Kobayashi and K. Nomizu, Foundations of Differential Geometry (John Wiley, New York 1963).

[10] C. N. Yang in Schrodinger: Centenary Celebration of a Polymath, edited by C. W. Kilmster (CUP, 1987).

[11] E. Schrodinger, Z. f. Phys. 12, 13 (1922).

[12] C. N. Yang, Phys. Rev. Lett. 33, 445 (1974).

[13] J. Anandan, Phys. Rev. D 53, 779 (1996).

[14] Y. Aharonov and D. Bohm, Phys. Rev. 115, 485 (1959).

[15] J. Anandan, Phys. Lett. A 195, 284 (1994).

[16] See, for example, reference 7, p. 244.

[17] J. Stachel in Proceedings of the Fourth Marcel Grossmann Meeting on General Relativity, R. Ruffini, ed. (Elsevier, Amsterdam, !986), p.1857-1862; J. Stachel in General Relativity and Gavitation - Proceedings of the 11th International Conference on General Relativity and Gravitation, M. A. H. MacCallum, ed. (Cambridge Univ. Press, 1987), p. 200-208; J. Stachel in Einstein Studies, vol. 1: Einstein and the History of General Relativity, D. Howard and J. Stachel, eds. (Birkhauser, Boston-Basel-Berlin, 1989), p. 63-100. See also R. Torretti, Relativity and Geometry (Pergamon Press, Oxford 1983), 5.6.

[18] A. Einstein and M. Grossmann, Zeitschr. Math. und Phys. 62, 225 (1913).

[19] H. R. Brown, International Studies in the Philosophy of Science, vol 9, 235-253, (1995).

[20] Y. Aharonov and L. Susskind, Phys. Rev. 158, 1237 (1967).

[21] Y. Aharonov and J. Anandan, in preparation.

[22] J. Anandan, J. of Gen. Rel. and Grav. 26, 125 (1994).

[23] Y. Aharonov and J. Anandan, Phys. Lett. A 160, 493 (1991); J. Anandan, Phys. Lett. A 164, 369 (1992). 
[24] P.G. Bergmann, Basic Theories of Physics, vol. 1, preface (Prentice-Hall, New York, 1949). 